\section{Expanding our ethnic understanding of the genome}

\section{see page 1007}

Providing a reminder that genomics research must make more of an effort to include ethnic variation, a research team from South Korea reports on actionable findings based on exome data from 196 individuals of Korean origin. This first report of potentially action-

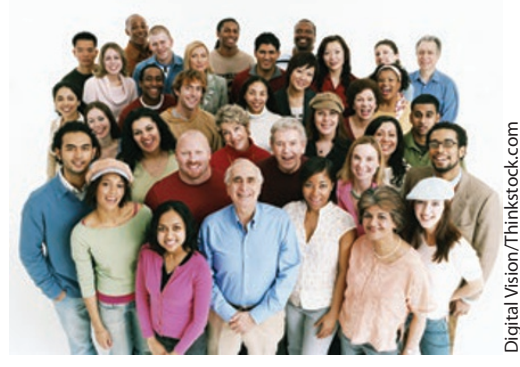
able secondary findings in an Asian population applies ACMG evidence-based guidelines to classify the variants. Secondary findings in the Korean study population (7\%) falls within the range of previously published studies in European and African subjects. For example, the 1000 Genomes Project found secondary findings in up to $11 \%$ of study subjects. Moreover, individuals in the healthy control group exhibited a proportion of pathogenic or likely pathogenic secondary variants (7\%) statistically equivalent to that in individuals in the study group who were suspected of having a genetic disease (6\%). The authors point out that while the ACMG guidelines for medically actionable genes are useful, they are not fully inclusive. For example, the ACMG list of 56 actionable genes does not include $C D H 1$, which is the causative gene for hereditary diffuse gastric cancer and is of particular interest in the Asian population because of its high prevalence in that group. The study highlights the need to more fully explore the genetic diversity found in ethnic populations. Currently, genomic studies skew toward western Europeans, making any conclusions drawn from them inherently limited in scope and leaving other ethnic populations at a disadvantage in efforts to personalize medicine. -Karyn Hede, News Editor

\section{IDIOM, a model program for rare-disease diagnosis}

\section{see page 995}

While many rare diseases are suspected to be of genetic origin, relatively few have been linked to a specific genetic origin.

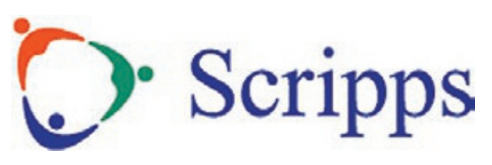
For patients and families, lack of diagnosis often leads to frustration. Now, a novel program modeled after the National Institutes of Health Undiagnosed Diseases Program is helping at least some individuals end their diagnostic odysseys. In this issue, the Scripps Idiopathic Diseases of Man (IDIOM) project reports on its first three years of operation. Of 121 cases reviewed for possible inclusion, 17 were ultimately selected for whole-genome sequencing. Of these, investigators were able to make a plausible molecular diagnosis in 10 cases. Three cases resulted in a confirmed molecular diagnosis. Of the confirmed cases, two led to identification of new gene-disease relationships. A third confirmed case was linked to a previously described but not fully characterized disorder. In all three cases, knowledge of the underlying genetic origin led to changes in treatment that had clinical benefit for the patients. The results suggest that genome sequencing could prove as useful for diagnosis of people with previously undescribed diseases as it is for diagnosis of known classic Mendelian disorders. The authors believe that IDIOM could provide a model for "application of genome sequencing across all rare genetic disorders, known and unknown." - Karyn Hede, News Editor

\title{
NEWS BRIEFS
}

\section{Sparing breast cancer patients chemotherapy}

More women who are diagnosed with breast cancer may be spared chemotherapy, based on a somatic gene expression panel that has now been proven to identify women at low risk of recurrence. The study was published in September 2015 in the New England Journal of Medicine. In the international clinical trial, 10,253 women diagnosed with hormone receptor-positive, HER2-negative cancer that had not spread to lymph nodes had their tumor tested for an expression

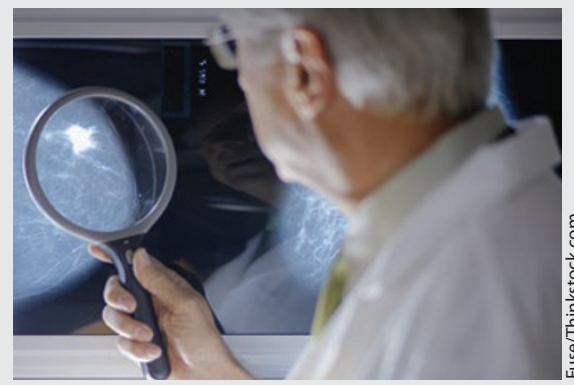

panel of 21 genes. After assessing the expression level of each gene, the test returns a score between 0 and 100 . The lower the score, the lower the chance the cancer will recur. In the trial, $15.9 \%$ of the women had a multigene test score of 10 or lower and received standard hormone therapy such as tamoxifen but did not undergo chemotherapy. An additional $68 \%$ of patients scored 11 to 25 . These women were randomly assigned to receive either hormone therapy plus chemotherapy or hormone therapy alone. Continued follow-up will be needed to determine whether women in the group with mid-range scores can also safely forgo chemotherapy. However, after five years, less than $2 \%$ of the patients 


\section{NEWS BRIEFS (continued)}

in this group had had a recurrence. The five-year overall patient survival was $98 \%$. These findings, the researchers concluded, provide the highest level of evidence yet that a multigene expression panel can spare the use of chemotherapy in women with low-scoring tumors. - Karyn Hede, News Editor

\section{Genomics of menopause}

We have now tripled the number of genes known to be associated with the age a woman undergoes menopause, thanks to a new large-scale genomic analysis published in Nature Genetics. In European populations, menopause occurs on average at age 51 but can vary by a decade or more in either direction. Risk of infertility and development of diseases such as breast cancer have been known to be associated with the timing of natural

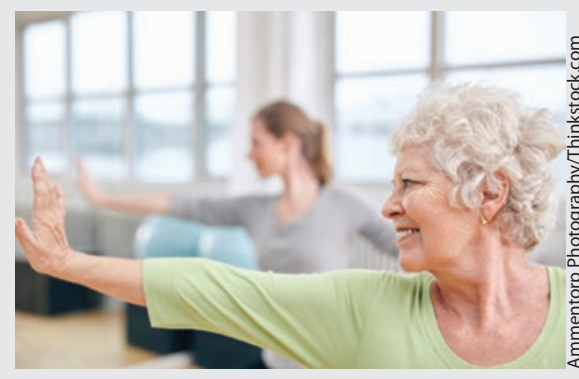

menopause, but little information has been available about how genetics affects both menopause timing and subsequent disease risk. Previous genome-wide association studies identified gene variants that explained only a tiny fraction of the natural variation in age of menopause. The new study included low-frequency coding variants that produced substantially more significant associations. These include links between reproductive aging ह and hypothalamic signaling, as well as DNA repair. The research team studied 70,000 women to identify common gene variants and identified 44 regions, including two regions with rare missense alleles that have a large effect: DNA helicase $B$ (encoded by $H E L B$ ), a DNA helicase that unwinds DNA during replication, transcription, repair and recombination, and SLCO4A1 (solute carrier organic anion transporter family, member 4A1), which transports organic molecules such as thyroid hormones. Pathway analyses identified DNA damage-response genes, including the first common coding variant in BRCA1 associated with any complex trait. Taken together, the findings implicate a relatively narrow set of pathways, with a large role for major known forms of DNA repair, determining the age of menopause. -Karyn Hede, News Editor 